\title{
THE SPLINE FINITE-STRIP METHOD IN THE BUCKLING ANALYSES OF THIN-WALLED STRUCTURES
}

\author{
G. M. VAN ERP \\ AND \\ C. M MENKEN \\ Eindhoven University of Technology, Den Dolech 2, 5612 AZ Eindhoven, The Netherlands
}

\begin{abstract}
SUMMARY
A spline finite-strip method capable of analysing the buckling of prismatic thin-walled structural members under arbitrary loading is presented. It utilizes cubic $\mathrm{B}_{3}$-splines as displacement functions in the longitudinal direction and polynomials in the transverse direction. Numerical integration is used to determine the geometric stiffness matrix of the strips. Two examples which demonstrate the accuracy and versatility of the method are presented.
\end{abstract}

\section{INTRODUCTION}

In the semi-analytical finite-strip method ${ }^{1}$ the displacement functions of a strip are expressed as products of interpolation polynomials in transverse direction and Fourier series functions in longitudinal direction, with the latter satisfying a priori the boundary conditions of the strip. This method has proved to be accurate and efficient for analysing the buckling of compressed structural members, but it has some disadvantages for analysing the buckling of beams loaded in bending and/or shear:

(i) Difficulties are experienced when dealing with non-periodic buckling modes (e.g. due to concentrated loads).

(ii) the buckling analysis of plate assemblies loaded in shear is problematic. ${ }^{2}$

(iii) Thin-walled beams may have many possible buckling modes, and since it is not known in advance which mode is critical, it will be necessary to solve the same eigenvalue problem for different series of Fourier terms in order to obtain the minimum buckling load. Especially in the case of beams with complex cross-sections, this repetitive process may be time-consuming.

The buckling model obtained by replacing the Fourier series functions by splines ${ }^{3-5}$ does not suffer from the problems mentioned above, and therefore seems more suitable to study the distortional and local buckling of beams under arbitrary loading. However, the aforementioned approaches are restricted to simple pre-buckling stresses. An extension of the range of applicability by using pre-buckling stresses calculated with the same displacement model as used for evaluating the critical stress level is discussed in this paper. 


\section{$B_{3}$-SPLINE APPROXIMATION}

The spline function chosen here to represent the displacements in the longitudinal direction is the basic cubic $B_{3}$-spline of equal section length. The displacement is taken as the summation of $(m+3)$ local $\mathrm{B}_{3}$-splines by

$$
g(x)=\sum_{i=-1}^{m+1} \alpha_{i} \psi_{i}(x)
$$

where $\psi_{i}(x)$ represents the locally hill-shaped $\mathrm{B}_{3}$-splines as shown in Figure $1(\mathrm{a})$ and $\alpha_{i}$ is a coefficient to be determined. The length $L$ of the structure is divided into $m$ equal-length sections as shown in Figure $1(\mathrm{~b})$. A local $\mathrm{B}_{3}$-spline is defined by

$$
\psi_{i}=\frac{1}{6 h^{3}}\left\{\begin{array}{lr}
0 & x<x_{i-2} \\
\left(x-x_{i-2}\right)^{3} & x_{i-2} \leqslant x \leqslant x_{i-1} \\
h^{3}+3 h^{2}\left(x-x_{i-1}\right)+3 h\left(x-x_{i-1}\right)^{2}-3\left(x-x_{i-1}\right)^{3} & x_{i-1} \leqslant x \leqslant x_{i} \\
h^{3}+3 h^{2}\left(x_{i+1}-x\right)+3 h\left(x_{i+1}-x\right)^{2}-3\left(x_{i+1}-x\right)^{3} & x_{i} \leqslant x \leqslant x_{i+1} \\
\left(x_{i+2}-x\right)^{3} & x_{i+1} \leqslant x \leqslant x_{i+2} \\
0 & x_{i+2}<x
\end{array}\right.
$$

The values of $\psi_{i}$ and $\psi_{i}$ at the section knots are shown in Table I.
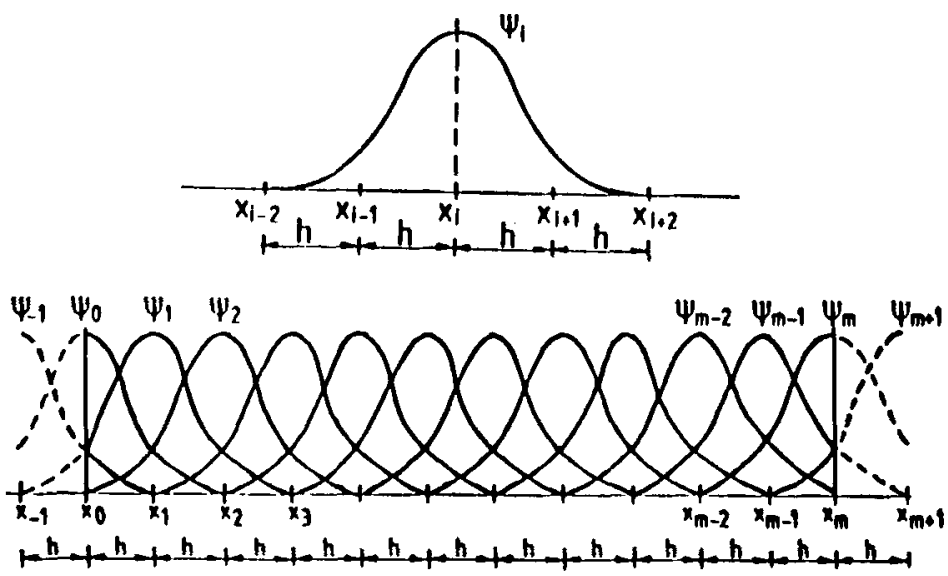

Figure 1. (a) basic cubic $\mathrm{B}_{3}$-spline; (b) a linear combination of $\mathrm{B}_{3}$-splines

Table I. The values of $\psi_{i}$ and $\psi_{i}^{\prime}$ at the section knots

\begin{tabular}{cccccc}
\hline & $x=x_{i-2}$ & $x=x_{i-1}$ & $x=x_{i}$ & $x=x_{i+1}$ & $x=x_{i+2}$ \\
\hline$\psi_{i}(x)$ & 0 & $1 / 6$ & $4 / 6$ & $1 / 6$ & 0 \\
$\psi_{i}(x)$ & 0 & $1 / 2 h$ & 0 & $-1 / 2 h$ & 0 \\
\hline
\end{tabular}




\section{MODIFICATION FOR BOUNDARY CONDITIONS}

There are many different methods for modifying the local boundary splines in order to satisfy the prescribed boundary conditions. In this paper a form is presented which is considered to be the most suitable in a finite-strip environment.

The knot coefficients $\alpha_{i-1}, \alpha_{i}$ and $\alpha_{i+1}$ are strongly related to the function values $g\left(x_{i}\right)$ and $g^{\prime}\left(x_{i}\right)$ (see Table I.):

$$
g\left(x_{i}\right)=\frac{1}{6} \alpha_{i-1}+\frac{4}{6} \alpha_{i}+\frac{1}{6} \alpha_{i+1} \quad \text { and } \quad g^{\prime}\left(x_{i}\right)=-\frac{1}{2 h} \alpha_{i-1}+\frac{1}{2 h} \alpha_{i+1}
$$

With (3) it is possible to replace the variables $\alpha_{-1}, \alpha_{0}$ and $\alpha_{m}, \alpha_{m+1}$ in (1) by the function value and first derivative of $g(x)$ at $x=0$ and $x=L$ :

$$
g(x)=g^{\prime}\left(x_{0}\right) \bar{\psi}_{-1}+g\left(x_{0}\right) \bar{\psi}_{0}+\alpha_{1} \bar{\psi}_{1}+\sum_{i=2}^{m-2} \alpha_{i} \psi_{i}+\alpha_{m-1} \bar{\psi}_{m-1}+g\left(x_{L}\right) \bar{\psi}_{m}+g^{\prime}\left(x_{L}\right) \bar{\psi}_{m+1}
$$

where

$$
\begin{aligned}
\bar{\psi}_{-1} & =-2 h \psi_{-1}+\frac{1}{2} h \psi_{0} & \bar{\psi}_{m-1} & =\psi_{m-1}-\frac{1}{2} \psi_{m}+\psi_{m+1} \\
\bar{\psi}_{0} & =\frac{6}{4} \psi_{0} & \bar{\psi}_{m} & =\frac{6}{4} \psi_{m} \\
\bar{\psi}_{1} & =\psi_{-1}-\frac{1}{2} \psi_{0}+\psi_{1} & \bar{\psi}_{m+1} & =-\frac{1}{2} h \psi_{m}+2 h \psi_{m+1}
\end{aligned}
$$

Now, with $g\left(x_{0}\right), g^{\prime}\left(x_{0}\right)$ and $g\left(x_{L}\right), g^{\prime}\left(x_{L}\right)$ (instead of $\alpha_{-1}, \alpha_{0}, \alpha_{m}$ and $\left.\alpha_{m+1}\right)$ as variables, the incorporation of prescribed kinematic boundary conditions at $x=0$ and $x=L$ into the $\mathrm{B}_{3}$-spline interpolation is straightforward.

\section{SPLINE FINITE-STRIP APPROACH}

The thin-walled member is discretized into a finite number of strips using $n$ nodal lines, which are further subdivided longitudinally into $m$ sections using $m+1$ section knots. Each section knot has four degrees of freedom which are related to the two out-of-plane deformations $w$ and $\theta$, and the two in-plane displacements $u$ and $v$. The nodal lines and section knot coefficients for a strip are shown in Figure 2 . Each nodal line has two additional knots outside the length of the strip in order to fully define the $B_{3}$-spline function over the length of the strip.

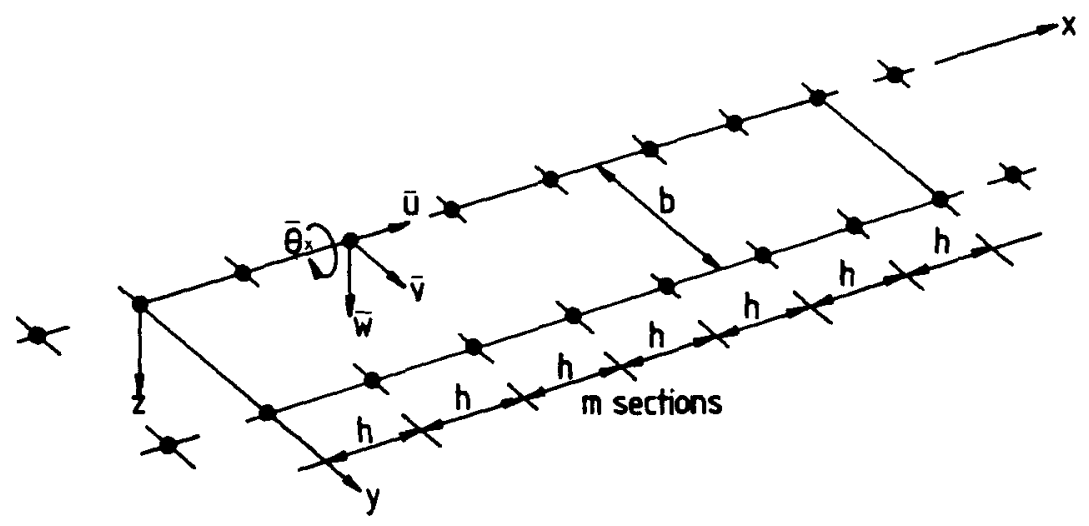

Figure 2. Strip with section knot coefficients 


\section{DISPLACEMENT FUNCTIONS}

The displacement functions of a strip are expressed as products of the longitudinal equidistant $\mathrm{B}_{3}$-splines and transverse polynomials as follows:

$$
\begin{aligned}
u & =N_{1} \psi^{\mathrm{T}} \overline{\mathbf{u}}_{i}+N_{2} \psi^{\mathrm{T}} \overline{\mathbf{u}}_{j} \\
v & =N_{1} \psi_{\mathrm{T}} \overline{\mathbf{v}}_{i}+N_{2} \psi^{\mathrm{T}} \overline{\mathbf{v}}_{j} \\
w & =N_{3} \psi^{\mathrm{T}} \overline{\mathbf{w}}_{i}+N_{4} \psi^{\mathrm{T}} \overline{\boldsymbol{\theta}}_{i}+N_{5} \psi^{\mathrm{T}} \overline{\mathbf{w}}_{j}+N_{6} \psi^{\mathrm{T}} \overline{\boldsymbol{\theta}}_{j}
\end{aligned}
$$

where:

$$
\begin{array}{ll}
N_{1}=1-\tilde{y} & N_{4}=y\left(1-2 \tilde{y}+\tilde{y}^{2}\right) \\
N_{2}=\tilde{y} & N_{5}=3 \tilde{y}^{2}-2 \tilde{y}^{3} \\
N_{3}=1-3 \tilde{y}^{2}+2 \tilde{y}^{3} & N_{6}=y\left(\tilde{y}^{2}-\tilde{y}\right) \\
\tilde{y}=\frac{y}{b} &
\end{array}
$$

$\psi$ represents columns with $\mathrm{B}_{3}$-splines and $\overline{\mathbf{u}}_{i}, \ldots, \overline{\boldsymbol{\theta}}_{j}$ are the displacement parameter columns for the nodal lines $i$ and $j$, respectively:

$$
\overline{\mathbf{u}}_{i}=\left[u^{\prime}\left(x_{0}\right), u\left(x_{0}\right), \bar{u}_{1}, \bar{u}_{2}, \ldots, \bar{u}_{m-2}, \bar{u}_{m-1}, u\left(x_{L}\right), u^{\prime}\left(x_{L}\right)\right]_{i}^{\mathrm{T}}
$$

where $u$ represents the real displacement, while $\tilde{u}_{i}$ represents a section knot coefficient (see equation 4).

\section{BUCKLING MODEL}

The linear buckling model considered here consists of two parts:

(i) The construction of a linear equlibrium model in order to evaluate the pre-buckling stresses.

(ii) The construction of a linear buckling or eigenvalue problem in order to evaluate the critical stress level.

\section{The equilibrium model}

For a structure in equilibrium, the frst variation of the total potential energy is zero. This property results in the following linear system of equations:

$$
\mathbf{K u}=\mathbf{f}
$$

where $\mathbf{K}$ is the gobal stiffness matrix, $\mathbf{u}$ represents the unknown displacement vector and $\mathbf{f}$ is the load vector. The solution of (7) defines the reference stress distribution over the domain.

\section{The buckling model}

The critical stresses and the associated buckling modes can be obtained by requiring the second variation of the potential energy to be stationary. The equivalent finite-strip model may be written in the following form:

$$
(\mathbf{K}+\lambda \mathbf{G}) \mathbf{v}=\mathbf{0}
$$


where $\mathbf{G}$ is the geometric stiffness matrix, which depends on the pre-buckling stress distribution, $\lambda$ is the buckling load parameter and $\mathbf{v}$ is the eigenvector.

\section{SOLUTION PROCESS}

At the strip level, the matrix $\mathbf{K}$ and vector $\mathbf{f}$ are worked out analytically. The resulting system of linear equations is solved using a skyline algorithm of Gauss decomposition. Once the displacements due to the reference load are known, the reference stresses can be determined. Because arbitrary loading conditions are considered, numerical integration is used to determine the geometric stiffness matrix G. Each integration over the area of a strip is subdivided into $m$ integrations, one over each section of length $h$. A $3 \times 3$ Gauss quadrature is used for each section. The eigenvalues and eigenvectors of the resulting eigenvalue problem are obtained using a subspace iteration procedure.

\section{NUMERICAL EXAMPLES}

The spline finite-strip buckling model described in this paper is capable of solving many of the bifurcation problems associated with thin-walled plate assemblies. An extensive treatise on its applications is beyond the scope of this paper, and therefore the method is illustrated by only two examples.

The accuracy of the solution is governed by the number of strips and sections used in the analysis; their influence is also evaluated.

\section{Plate strip loaded in pure shear}

The first example to be considered is that of a plate strip loaded in pure shear. The geometry and loading conditions are shown in Figure 3. The buckling coefficient of the present plate strip equals $5 \cdot 38 .{ }^{6}$ The numerical results are presented in Table II and a contour plot of the out-of-plane displacements, obtained with five strips and 30 sections, is shown in Figure 4 . For the sake of clarity both the strip and section subdivision are shown.

\section{T-beam loaded by a concentrated force}

In the second example, the buckling behaviour of a T-beam loaded by a concentrated force at mid-length is evaluated. In addition to the global buckling mode, the local mode with the lowest critical load is also considered. The geometry, loading condition and strip configuration are shown in Figure 5. The numerical results obtained with a different number of sections and 15 strips are presented in Table III. The buckling modes determined with 30 sections are shown in Figures 6 and 7.

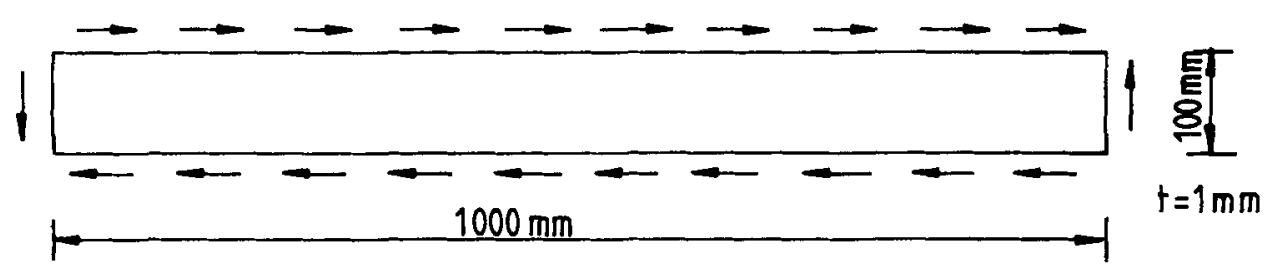

Figure 3. Plate strip loaded in pure shear 
Table II. Buckling coefficients of a plate strip in pure shear

\begin{tabular}{|c|c|c|c|c|}
\hline \multirow{2}{*}{$\begin{array}{l}\text { No. of } \\
\text { strips }\end{array}$} & \multicolumn{3}{|c|}{$\begin{array}{l}\text { Numerical buckl. coeff. } \\
\text { No. of sections }\end{array}$} & \multirow{2}{*}{$\begin{array}{l}\text { Analytical } \\
\text { buckl. coeff }\end{array}$} \\
\hline & 10 & 20 & 30 & \\
\hline 5 & $5 \cdot 67$ & $5 \cdot 40$ & $5 \cdot 39$ & \multirow{2}{*}{$5 \cdot 38$} \\
\hline 10 & $5 \cdot 65$ & $5 \cdot 39$ & $5 \cdot 38$ & \\
\hline
\end{tabular}

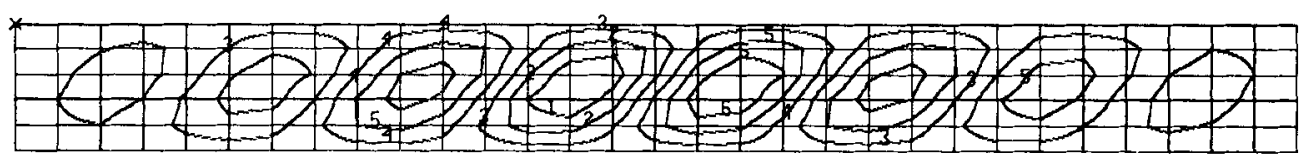

Figure 4. Contour plot of the out-of-plane displacements of a plate strip in pure shear

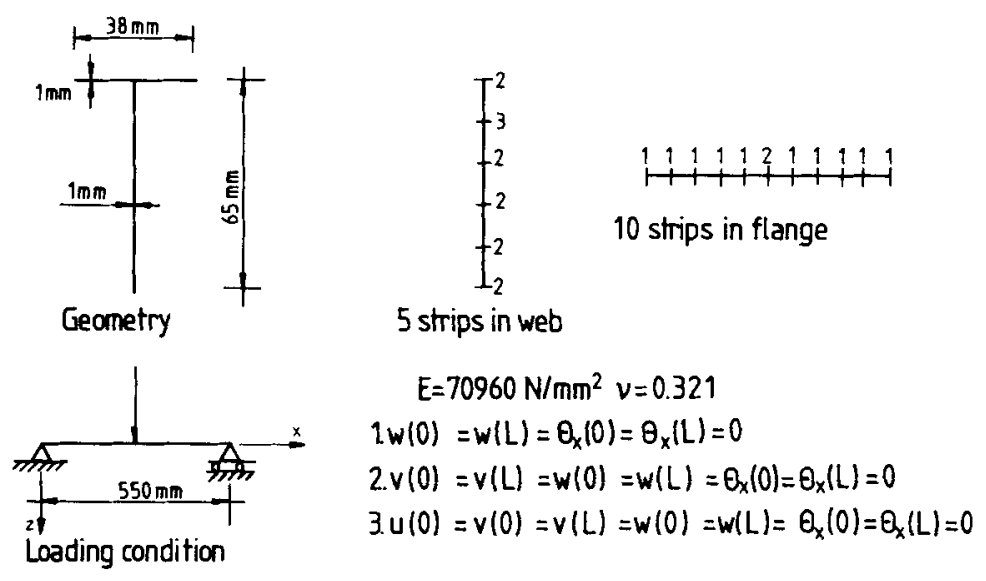

Figure 5. Geometry and loading conditions of the T-beam

Table III. Buckling loads of the T-beam

\begin{tabular}{cccc}
\hline $\begin{array}{c}\text { No. of } \\
\text { strips }\end{array}$ & $\begin{array}{c}\text { No. of } \\
\text { sections }\end{array}$ & $\begin{array}{c}\text { 1st buckling load } \\
\times 10^{3} \mathrm{~N} \\
\text { Numerical }\end{array}$ & $\begin{array}{c}\text { 2nd buckling load } \\
\times 10^{3} \mathrm{~N} \\
\text { Numerical }\end{array}$ \\
\hline \multirow{3}{*}{15} & 10 & $1 \cdot 88$ & $2 \cdot 72$ \\
& 20 & 1.87 & $2 \cdot 48$ \\
& 30 & 1.87 & $2 \cdot 45$ \\
\hline
\end{tabular}




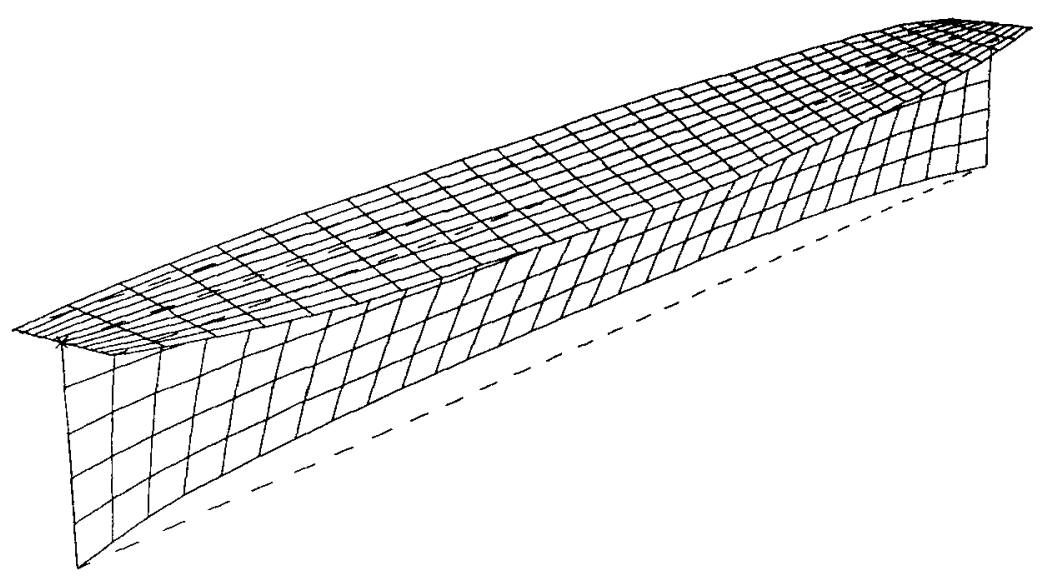

Figure 6. First buckling mode of the T-beam

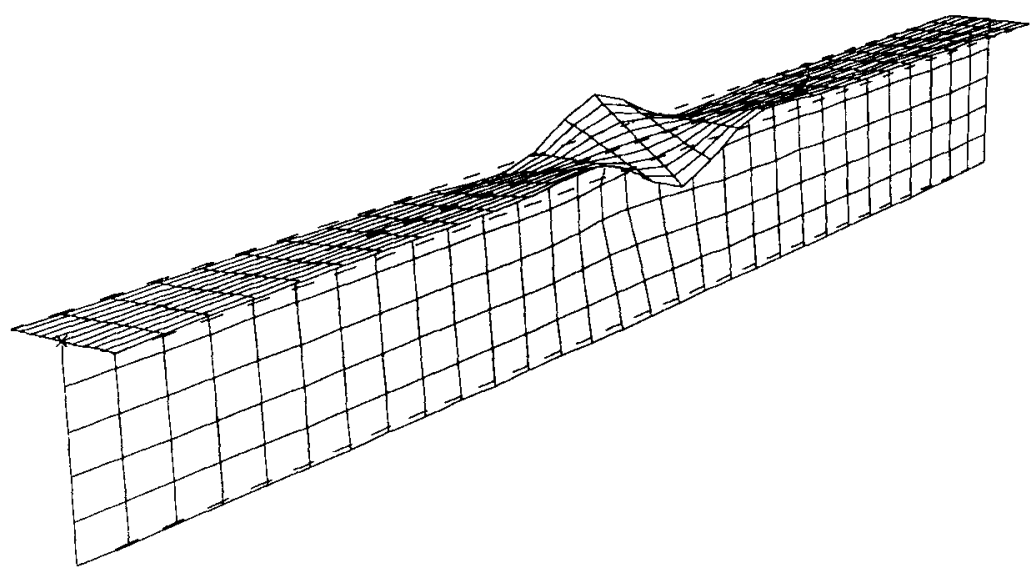

Figure 7. Second buckling mode of the T-beam

The good performance of the present approach for other differently loaded structures is demonstrated by Van Erp. ${ }^{7}$

\section{CONCLUSIONS}

The previous examples demonstrate the accuracy and versatility of the present approach for predicting the buckling loads of thin-walled plate assemblies under arbitrary loading. The simplicity of the semi-analytical finite-strip method is preserved, while the problems of dealing with non-periodic buckling modes, shear and non-simple support are eliminated. The number of degrees of freedom required for a spline finite-strip analysis is considerably larger than for the semi-analytical finite-strip method, but it is still approximately $40 \%$ smaller than that of a comparable finite-element analysis.

The combination in the longitudinal direction of strips with different section lengths can easily be accomplished. Extending the present computer program with this option will result in a reduction of the computer time for structures with concentrated short-wave local buckles. 
The present approach uses a $3 \times 3$ Gauss quadrature per section length to construct the geometric stiffness matrix. However, orientating calculations indicate that a lower-order Gauss quadrature also leads to satisfactory results.

\section{REFERENCES}

1. Y. K. Cheung, Finite Strip Method in Structural Analysis, Pergamon Press, London, 1976.

2. M. Mahendran and N. W. Murray, 'Elastic buckling analyses of ideal thin-walled structures under combined loading using a finite strip method', Thin-Walled Structures, 4, 329-462 (1986).

3. S. C. Fan, Y. K. Cheung and C. Q. Wu, 'Spline finite strip in structural analysis', Proceedings of the International Conference on Finite Element Methods, 704-709, 1982.

4. S. C. Fan and Y. K. Cheung, 'Analysis of shallow shells by spline finite strip method', Eng. Struct., 5 (4), 255-263 (1983).

5. S. C. W. Lau and G. J. Hancock, 'Buckling of thin flat-walled structures by a spline finite strip method', Thin-Walled Struct., 4, 269-294 (1986).

6. H. G. Allen and P. S. Bulson, Background to Buckling, McGraw-Hill Book Company (UK) Limited, 1980.

7. G. M. van Erp, 'Advanced buckling analyses of beams with arbitrary cross sections' Ph.D. thesis 1989, Eindhoven University of Technology, The Netherlands. 